\title{
Clusters and the entropy in opinion dynamics on complex networks
}

\author{
Wenchen Han \\ College of Physics and Electronic Engineering, Sichuan Normal University, Chengdu, 610101, China \\ Yuee Feng \\ College of Arts and Sciences, Jiangsu Aviation Technical College, Zhenjiang, 212134, China \\ Xiaolan Qian \\ School of Media and Engineering, Communication University of Zhejiang, Hangzhou, 310018, China \\ Qihui Yang \\ Department of Electrical and Computer Engineering, \\ Kansas State University, Manhattan, Kansas 66506, USA \\ Changwei Huang* \\ School of Computer, Electronics and Information, Guangxi University, Nanning, 530004, China
}

\begin{abstract}
In this work, we investigate a heterogeneous population in the modified Hegselmann-Krause opinion model on complex networks. We introduce the Shannon information entropy about all relative opinion clusters to characterize the cluster profile in the final configuration. Independent of network structures, there exists the optimal stubbornness of one subpopulation for the largest number of clusters and the highest entropy. Besides, there is the optimal bounded confidence (or subpopulation ratio) of one subpopulation for the smallest number of clusters and the lowest entropy. However, network structures affect cluster profiles indeed. A large average degree favors consensus for making different networks more similar with complete graphs. The network size has limited impact on cluster profiles of heterogeneous populations on scale-free networks but has significant effects upon those on small-world networks.
\end{abstract}

\section{INTRODUCTION}

The field of multiagent network dynamics, which can reveal the emergence of collective behavior characteristics based on local individual interaction rules, has been investigated by researchers from a vast range of disciplines in recent years 1 1 3]. Agents' opinions can change in time subject to the interactions between the neighboring agents as well as the global feedback and external factors. The opinion dynamics characterized with statistical physics has been developed in the social opinion research. Opinion dynamics models are classified into discrete opinion dynamics models and continuous ones. More specifically, opinions are modeled as variables, discrete or continuous. In the discrete case, binary opinion models are most studied in terms of their analogy with spin systems. Well-known discrete opinion dynamics models include the voter model [4], the Sznajd model [5], the Galam majority-rule model [6], and the nonconsensus opinion model [7, 8]. Among the continuous opinion dynamics models, the Deffuant-Weisbuch model [9] and the Hegselmann-Krause (HK) model [10] have attracted much attention. In the final stationary state, opinion clusters can be one (consensus), two (polarization), or many (fragmentation).

Opinions are continuous variables in the interval $[0,1]$

*Electronic address: cwhuang@gxu.edu.cn in an HK model. The evolution is treated as a series of discrete time steps. At each time step, an agent updates her opinion by adopting the average of her neighbors' opinions and her own. Two agents are neighbors only when the difference of their opinions is below the threshold, i.e., the bounded confidence. In a homogeneous population, where agents are with a same set of parameters, the consensus is the result of agents with a high bounded confidence, while a fragmentation is caused by a low bounded confidence. On the other hand, agents with heterogeneous bounded confidences could help agents to reach consensus [11, 12]. Fu et al. found that agents with high bounded confidence could not contribute to forging consensus and might lead to fragmentation [13]. While Han et al. [14] found whether agents would reach consensus depended upon not only the bounded confidence but also the stubbornness, which measured the extent of an agent's insistence on her own opinion [15 18].

The network structure provides a background for the opinion dynamics system, in which nodes stand for agents and links connecting the nodes represent the possible interactions between agents. The network structure can be a complete graph, a random network 19], a scale-free network 20], or a small-world network [21]. However, it is known that network structures affect dynamical processes on networks 22 24]. Results of complete graphs and square lattices are similar for large bounded confidence values, except for remaining a few extreme opinions 25]. Jalili studied the social power for the consensus in opinion dynamics on complex networks, found that giv- 
ing the social power to nodes in small-world networks cannot significantly affect the consensus while hubs with social power in scale-free networks can 26]. Even for small-world networks, directed networks and bi-directed networks show different effects on opinion dynamics 27]. Bagnoli and Rechtman studied a society with conformist and reasonable contrarian agents, and they found systems on small-world networks and scale-free networks showed some similarities due to the long-range connectivity 28, 29].

In this work, we considered a heterogeneous population on different complex networks, where agents in a same subpopulation shared a same set of parameters (i.e., bounded confidence and stubbornness) and different subpopulations were with different sets of parameters. We paid our attention to the cluster profile while varying one of the parameters in this heterogeneous population. The cluster profile contained the information about the number of opinion clusters and the relative sizes of all clusters, and it implied the Shannon information entropy about the sizes of clusters. We found that the cluster profile was the trade-off of competition effects of agent's parameters. Furthermore, we also investigated the effect of the average degree of networks and the effect of the network size on the cluster profile. A large average degree made networks behave like a complete graph, supporting fewer clusters, a larger size of the largest cluster, and a low entropy. Besides, the network size had less impact on cluster profiles of populations on scale-free networks but significant impacts on those on small-world networks, due to the strongly connected local structures.

\section{MODEL}

We consider a heterogeneous population composed of $N$ agents on complex networks. Agent $i$ is placed on the node $i$ in the network. $A_{i j}$ is an element of the adjacency matrix for the network structure. $A_{i j}=A_{j i}=1$ denotes node $i$ and node $j$ are connected and that agent $i$ and agent $j$ may be neighbors. $A_{i j}=A_{j i}=0$ indicates that node $i$ and node $j$ are disconnected and that agent $i$ and agent $j$ cannot be neighbors. Additionally, self-edges are avoided $\left(A_{i i}=0\right)$. The opinion of agent $i$ at discrete time $t$ is described by a continuous variable $x_{i}(t)$, satisfying $0 \leq x_{i}(t) \leq 1$. The evolution of the opinion of agent $i$ follows the updating rule

$x_{i}(t+1)= \begin{cases}\alpha_{i} x_{i}(t)+\frac{\left(1-\alpha_{i}\right)}{\left\|N_{i}(t)\right\|} \sum_{j \in N_{i}(t)} x_{j}(t), & \left\|N_{i}(t)\right\|>0, \\ x_{i}(t), & \left\|N_{i}(t)\right\|=0 .\end{cases}$

$\alpha_{i}$, which is in the interval $[0,1]$, describes the stubbornness of agent $i$, suggesting the extent of agent $i$ insisting on her own opinion. $\alpha_{i}=0$ means agent $i$ is a conformist, just following other neighbors' opinions, while agent $i$ with $\alpha_{i}=1$ is a zealot, only sticking to her own opinion and neglecting others [30]. Note that agent $i$ is more stubborn than agent $j$ when $\alpha_{i}$ is larger than $\alpha_{j}$. $N_{i}(t)$ for agent $i$ at time $t$ is the set of neighbor agents, whose opinions satisfy $\left|x_{j}(t)-x_{i}(t)\right|<\sigma_{i}$ and $A_{i j}=1$. $\left\|N_{i}(t)\right\|$ is the cardinality of $N_{i}(t)$. Bounded confidence $\sigma$ reflects the psychological concept of selective exposure, which refers to an individual's tendency to favor information that supports her opinion while neglecting conflicting arguments, with $\sigma \in(0,1]$. We name agents with high $\sigma$ as open-minded ones and agents with low $\sigma$ as close-minded ones.

In this work, the total population is composed of $M$ subpopulations. Each subpopulation with $\rho_{l} N$ agents, with the ratio $\rho_{l} \in[0,1]$ and $\sum_{l=1}^{l=M} \rho_{l}=1$, takes the bounded confidence $\sigma_{l}$ and the stubbornness $\alpha_{l}$. The subpopulation with $\sigma_{l}$ and $\alpha_{l}$ is denoted as $C_{l}$ for convenience. When opinions of all agents stop evolving, we say the system reaches its steady state. Then we monitor the number of opinion clusters, $N_{c}$, where connecting agents within a same cluster share a same opinion. Unconnected agents with a same opinion belong to different clusters. So it is with connected agents with different opinions. $S_{i}$ is the relative size of an opinion cluster, namely the ratio between the number of agents in the cluster and the number of agents in the system. The relative size of all opinion clusters can be sorted in a descending order, i.e., $1 \geq S_{1} \geq S_{2} \geq \cdots \geq S_{N_{c}}>0$. Additionally, $\left\{S_{1}, S_{2}, \cdots, S_{N_{c}}\right\}$ is an opinion cluster profile. We introduce the Shannon information entropy $H$ [31] as

$$
H=-\sum_{i=1}^{N_{c}} S_{i} \log _{2} S_{i}
$$

The Shannon information entropy, analogy with Boltzmann Entropy [28, 29], describes the information gain of a certain opinion cluster profile. When $N_{c}=1$ for the existence of only one cluster and $S_{1}=1$, no information can be gained $(H=0)$. If the opinions are scattered (e.g., $N_{c}=N$ and $S_{i}=1 / N$ for $\left.i=1,2, \cdots, N\right)$, much information can be gained $\left(H=\log _{2}(N)\right)$. For similar cluster sizes, a cluster profile with more clusters corresponds to a higher $H$. If two opinion cluster profiles share a same number of clusters, the profile composed of less heterogeneous clusters, where some clusters are with similar sizes, are with a higher $H$. In this work, we consider the connecting structure between agents as complex networks, including random networks (RNs), scale-free networks (SFNs), and small-world networks (SWNs) with rewiring possibility 0.1. All the networks are connected and have $N$ nodes and the $N K / 2$ links, where $K$ is the average degree. We consider the population size $N=1000$ and the average degree $K=20$ unless specified. The simulation results are averaged over 100 realizations with uniform random initial conditions in opinions. The opinions of agents are updated synchronously. 

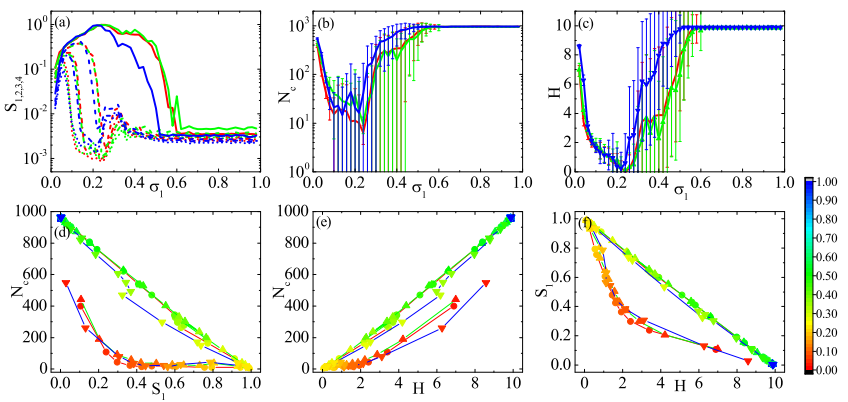

FIG. 1: The dependent relations for varying $\sigma_{1}$ when $\rho_{1}=0.9$, $\alpha_{1}=0.8, \rho_{2}=0.1, \alpha_{2}=0$, and $\sigma_{2}=0.04$. The red line/circle dots for random networks, the green line/up-triangles dots for scale-free networks, the blue line/down-triangles dots for small-world networks. (a) the dependence of four largest clusters $S_{1,2,3,4}$ (solid, dash, dash-dot, short dash lines) on $\sigma_{1}$. (b) the dependence of the number of clusters $N_{c}$ on $\sigma_{1}$. (c) the dependence of the Shannon information entropy $H$ on $\sigma_{1}$. (d) the relation of $N_{c}$ against $S_{1}$. (e) the relation of $N_{c}$ against $H$. (f) the relation of $S_{1}$ against $H$. In panels (d-f), the colors of dots for the values of $\sigma_{1}$ linearly.

\section{SIMULATION RESULTS}

In a homogeneous population $(M=1)$, the stubbornness $\alpha$ only affects the transition time when the bounded confidence $\sigma$ is fixed [32]. A larger $\alpha$ is for a longer transition process. Increasing $\sigma$ of agents always leads to a smaller number of opinion clusters $N_{c}$ and a larger size of the largest opinion cluster $S_{1}$ when the bounded confidence is smaller than the threshold $\left(\sigma_{c}=0.25\right)$. Beyond $\sigma_{c}$, only one opinion cluster exists. It is also known that the dependence of $N_{c}$ (or $S_{1}$ ) on $\sigma$ is caused by the competition effects of agents' bounded confidences and stubbornness in a heterogeneous population $(M \geq 2)$ on complete graphs 14.

In the following, we will report the results in a heterogeneous opinion population on complex networks (RNs, SFNs, SWNs) and pay attention to the cluster profile in the final configuration, which can be depicted by $N_{c}$, $S_{i}, i=1,2, \cdots, N_{c}$, and $H$. We consider a heterogeneous population just composed of two subpopulations $M=2$, where $\rho_{1} N$ agents are with $\sigma_{1}$ and $\alpha_{1}, \rho_{2} N$ agents are with $\sigma_{2}$ and $\alpha_{2}$, and $\rho_{1}+\rho_{2}=1$.

In Fig. 1, $C_{1}$ agents are more stubborn than closeminded $C_{2}$ agents $\left(\rho_{1}=0.9, \alpha_{1}=0.8, \rho_{2}=0.1, \alpha_{2}=0.0\right.$, and $\sigma_{2}=0.04$ ), where $C_{2}$ subpopulation tends to form about 10 clusters when only $C_{2}$ agents are on a complete graph. In Fig. 1 (a), increasing the bounded confidence of $C_{1}$ agents makes $S_{1}$ first increase, then decrease, and remain constant finally $\left(\sigma_{1}>0.6\right)$, while it makes $N_{c}$ decrease, increase, then remain constant, as shown in Fig. 1 (b). Interestingly, when the $C_{1}$ agents are much open-minded (e.g., $\sigma_{1}=0.8$ ), all clusters are small and the size of the largest opinion cluster is even smaller than that when $C_{1}$ agents are close-minded (e.g., $\sigma_{1}=0.1$ ).
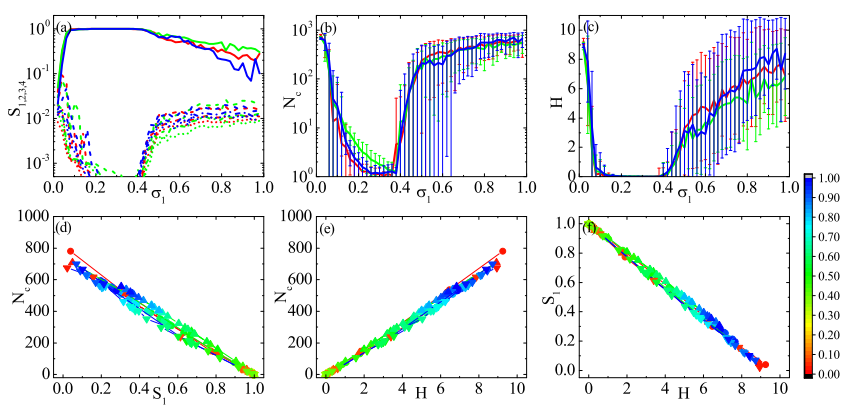

FIG. 2: The dependent relations for varying $\sigma_{1}$ when $\rho_{1}=0.9$, $\alpha_{1}=0, \rho_{2}=0.1, \alpha_{2}=0.8$, and $\sigma_{2}=0.3$. The red line/circle dots for random networks, the green line/up-triangles dots for scale-free networks, the blue line/down-triangles dots for small-world networks. (a) the dependence of four largest clusters $S_{1,2,3,4}$ (solid, dash, dash-dot, short dash lines) on $\sigma_{1}$. (b) the dependence of the number of clusters $N_{c}$ on $\sigma_{1}$. (c) the dependence of the Shannon information entropy $H$ on $\sigma_{1}$. (d) the relation of $N_{c}$ against $S_{1}$. (e) the relation of $N_{c}$ against $H$. (f) the relation of $S_{1}$ against $H$. In panels (d-f), the colors of dots for the values of $\sigma_{1}$ linearly.

A high $\sigma$ leads to fragmentation [13, 14]. However, in this case, this is caused by not only the competition effect between $\sigma$ and $\alpha$ but also the connection constrains of network structures, which can be verified in the following discussion. However, the dependence of $S_{1}$ on $\sigma_{1}$ and the dependence of $N_{c}$ on $\sigma_{1}$ are not simply negative correlated, as shown in Fig. 1 (d). For example, $S_{1}$ increases while $N_{c}$ almost remains when $0.1 \leq \sigma_{1} \leq 0.2$. This means that only considering $S_{1}$ and neglecting the evolution of other opinion clusters cannot explain the behavior of $N_{c}$, which counts up all clusters. That's because $S_{1}$ keeps increasing along with the decreases of $S_{4}, S_{3}$, and $S_{2}$ when $N_{c}$ almost remains. The Shannon information entropy is a good measure for taking all clusters into account. It encodes not only the number but also the heterogeneity of all opinion clusters. In Fig. 1 (c), $H$ decreases when $S_{1}$ increases along with $S_{4}, S_{3}, S_{2}$ decreasing for $\sigma_{1} \in(0.1,0.2)$. $H$ shows some similar features but not exactly the same with $N_{c}$, which is not a strict linear relationship as shown in Fig. 11(e). Figure 1 (f) shows the relation of $S_{1}$ against $H$ is a roughly negative linear one, suggesting that a same $H$ corresponds to different $S_{1}$ and vice versa. Figure 1 (d-f) suggests that one of $H, N_{c}$, and $S_{1}$ corresponds to different cluster profiles and that only one of $H, N_{c}$, and $S_{1}$ is not sufficient to describe a cluster profile. Additionally, Fig. 1 shows cluster profiles on different networks share many characteristics.

Figure 2 shows the results of $C_{1}$ agents $\left(\alpha_{1}=0.0\right)$ with stubborn but open-minded $C_{2}$ agents $\left(\alpha_{2}=0.8\right.$ and $\sigma_{2}=0.3$ ), where $C_{2}$ agents can achieve consensus when only $C_{2}$ agents exists on a complete graph. The dependence of $S_{1}, N_{c}$, and $H$ on $\sigma_{1}$ is qualitatively the same as that of the population in Fig. 11 regardless of dif- 

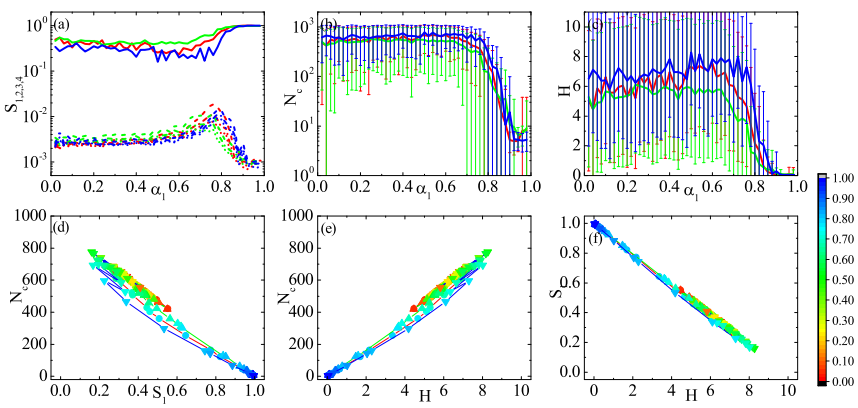

FIG. 3: The dependent relations for varying $\alpha_{1}$ when $\rho_{1}=$ $0.9, \sigma_{1}=0.3, \rho_{2}=0.1, \alpha_{2}=0$, and $\sigma_{2}=0.04$. The red line/circle dots for random networks, the green line/uptriangles dots for scale-free networks, the blue line/downtriangles dots for small-world networks. (a) the dependence of four largest clusters $S_{1,2,3,4}$ (solid, dash, dash-dot, short dash lines) on $\sigma_{1}$. (b) the dependence of the number of clusters $N_{c}$ on $\sigma_{1}$. (c) the dependence of the Shannon information entropy $H$ on $\sigma_{1}$. (d) the relation of $N_{c}$ against $S_{1}$. (e) the relation of $N_{c}$ against $H$. (f) the relation of $S_{1}$ against $H$. In panels (d-f), the colors of dots for the values of $\alpha_{1}$ linearly.

ferent values of $\alpha_{1}, \alpha_{2}$, and $\sigma_{2}$. However, it is a little different from the result when a heterogeneous population sitting on complete graphs [14], where increasing $\sigma_{1}$ always leads to consensus when the other subpopulation agents are open-minded. A steep decrease in $H$ suggests a quick process of clusters merging when $\sigma_{1} \in(0.02,0.1)$, presented by the largest cluster increasing with the vanishing of small clusters. However, further increasing $\sigma_{1}$ even leads to a smaller $S_{1}$, a larger $N_{c}$, and a higher $H$ in surprise, when $\sigma_{1}>0.4$, evolving on complex networks. These results are constrained by connections of network structures. Different from what is shown in Fig. 1 $N_{c}$ against $S_{1}$ (Fig. 2 (d)) and $S_{1}$ against $H$ (Fig. 2 (f)) exhibit highly negative linear relations and $N_{c}$ against $H$ (Fig. 2 (e)) is with a highly linear relation. These high correlations imply that one of $H, N_{c}$, and $S_{1}$ is enough to describe a cluster profile, in this case. Moreover, the dependent relations show no difference between populations on networks.

By varying $\alpha_{1}$, cluster profiles for open-minded agents $\left(\sigma_{1}=0.3\right)$ interacting with not stubborn and closeminded agents $\left(\sigma_{2}=0.04, \alpha_{2}=0\right)$ are shown in Fig. 3. Slightly different from what happens on complete graphs, where increasing the stubbornness of open-minded agents always leads to smaller clusters and a larger largest cluster [14], on complex networks $S_{1}$ decreases then increases while $N_{c}$ and $H$ increases then decreases when $\alpha_{1}$ increases. When $C_{1}$ agents are not so stubborn, the decrease of $S_{1}$ is caused by the local equilibrium, where $C_{1}$ agents quickly adjust their opinions to the average opinion of their neighbors. While $C_{1}$ are very stubborn, they wait their neighbors to adjust their opinions, which favors the cluster merging process and leads to a larger largest clusters. We have also checked the results of some other
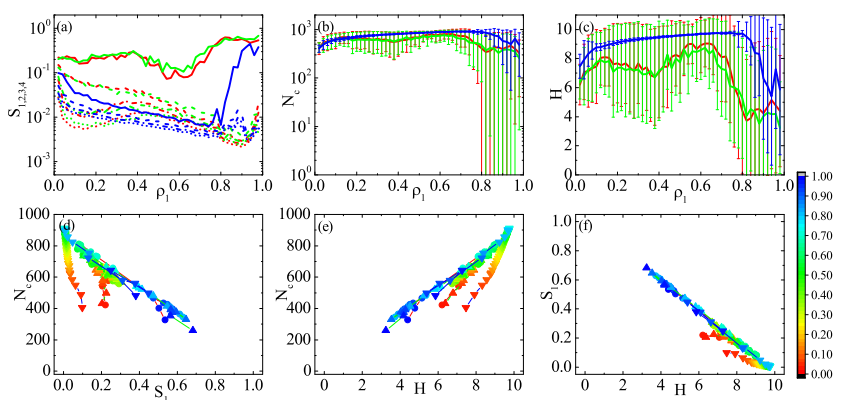

FIG. 4: The dependent relations for varying $\rho_{1}$ when $\alpha_{1}=0$, $\sigma_{1}=0.3, \rho_{2}=1-\rho_{1}, \alpha_{2}=0.8$, and $\sigma_{2}=0.04$. The red line/circle dots for random networks, the green line/uptriangles dots for scale-free networks, the blue line/downtriangles dots for small-world networks. (a) the dependence of four largest clusters $S_{1,2,3,4}$ (solid, dash, dash-dot, short dash lines) on $\sigma_{1}$. (b) the dependence of the number of clusters $N_{c}$ on $\sigma_{1}$. (c) the dependence of the Shannon information entropy $H$ on $\sigma_{1}$. (d) the relation of $N_{c}$ against $S_{1}$. (e) the relation of $N_{c}$ against $H$. (f) the relation of $S_{1}$ against $H$. In panels (d-f), the colors of dots for the values of $\rho_{1}$ linearly.

heterogeneous population. The (negative) linear relation of $N_{c}$ against $H$ ( $N_{c}$ against $S_{1}$ or $S_{1}$ against $H$ ) is robust. It can be inferred that one of $H, N_{c}$, and $S_{1}$ is sufficient for describing cluster profiles when varying the stubbornness of one subpopulation. Furthermore, the dependent relations on $\alpha_{1}$ is almost independent of network structures.

The ratios of subpopulations affect the influence of the

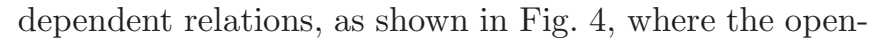
minded subpopulation $C_{1}\left(\alpha_{1}=0, \sigma_{1}=0.3\right)$ is with a more stubborn close-minded subpopulation $C_{2}\left(\alpha_{1}=0.8\right.$, $\left.\sigma_{1}=0.04\right)$. In this case, cluster profiles on different complex networks share some characteristics, while cluster profiles of populations on SWNs show some differences from those on RNs or SFNs. In Fig. 目 (a), when $\rho_{1}$ is rather small, the four largest clusters are with similar sizes in spite of network structures. Increasing the ratio of $C_{1}$ subpopulation $\rho_{1}$ when $\rho_{1}<0.8$, the size of the largest cluster $S_{1}$ almost remains along with other clusters shrinking on RNs or SFNs, while the largest cluster together with other clusters shrinks on SWNs. When $\rho_{1}$ further increases, $S_{1}$ continues to increase and it increases to around 0.5. Furthermore, the number of clusters $N_{c}$ when populations evolve on SWNs is comparable larger than that on RNs or SFNs in Fig. 4 (b). So it is with the entropy $H$ in Fig. 团(c). Although the roughly linear dependent relations remain, differences between populations on SWNs and those on RNs or SFNs are more obvious, as shown in Fig团(d-f). On SWNs, the population will show more clusters with smaller $S_{1}$, higher $H$, and larger $N_{c}$. This is caused by the strongly connected local structures, causing local equilibrium, in SWNs.

The results above show the effects of parameters of agents on cluster profiles of heterogeneous populations. 

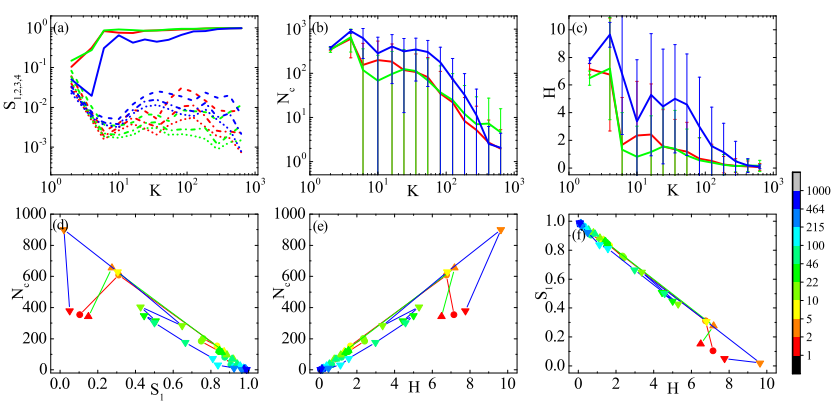

FIG. 5: The dependent relations for varying $K$ when $\rho_{1}=0.9$, $\alpha_{1}=0.8, \sigma_{1}=0.3, \rho_{2}=0.1, \alpha_{2}=0$, and $\sigma_{2}=0.04$, with the population size $N=1000$. The red line/circle dots for random networks, the green line/up-triangles dots for scale-free networks, the blue line/down-triangles dots for small-world networks. (a) the dependence of four largest clusters $S_{1,2,3,4}$ (solid, dash, dash-dot, short dash lines) on $\sigma_{1}$. (b) the dependence of the number of clusters $N_{c}$ on $\sigma_{1}$. (c) the dependence of the Shannon information entropy $H$ on $\sigma_{1}$. (d) the relation of $N_{c}$ against $S_{1}$. (e) the relation of $N_{c}$ against $H$. (f) the relation of $S_{1}$ against $H$. In panels (d-f), the colors of dots for the values of $K$ logarithmically.

It is convinced that cluster profiles are the trade-off of competition between all parameters [14] and that features of cluster profiles are almost independent of network structures when only one parameter of agents varies. However, network structures should have some effects on cluster profiles, as known in Ref. [22 24] and shown in Fig. 4. In the following, two parameters of network structures, the average degree $K$ and the network size $N$, are concerned.

We first study the influence of the average degree $K$ on these dependent relations. $S_{1}$ increases while $N_{c}$ and $H$ decrease when $K$ increases, as shown in Fig. $5(\mathrm{a}$-c). The difference between SWNs and RNs or SFNs still remains when $K$ is small, but it is vanishing when $K$ is rather large. It should be noticed that when $K$ increases with $N$ fixed the difference between different network structures vanishes. When the network connectivity is high $(K / N \rightarrow 1)$, all network structures will show the characteristics of complete graphs. The results in Fig. 5 (d-f) confirm this, with all dot-lines for different networks converging to a same point, which almost corresponds to the result for the population evolving on complete graphs.

We also investigate the effects of the network size $N$. In this case, we monitor the relative number of clusters $N_{c} / N$ in place of the absolute number of clusters $N_{c}$. Despite of network structures, $S_{1}$ is decreasing while $N_{c} / N$ and $H$ are increasing when $N$ increases. Thanks to the strongly connected local structures, the variation of $H$ on SWNs is more obvious than that on RNs or SFNs. Although the linear relations are robust, the differences between the networks are shown in Fig. 6 (d-f) directly. The increase of $N$ shows the least effect on SFNs and the most effects on SWNs, which suggests that effects of
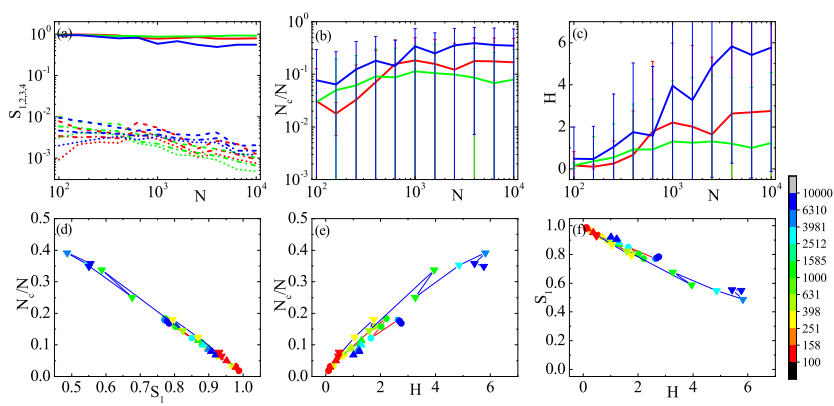

FIG. 6: The dependent relations for varying $N$ when $\rho_{1}=0.9$, $\alpha_{1}=0.8, \sigma_{1}=0.3, \rho_{2}=0.1, \alpha_{2}=0$, and $\sigma_{2}=0.04$, with the average degree $K=20$. The red line/circle dots for random networks, the green line/up-triangles dots for scale-free networks, the blue line/down-triangles dots for small-world networks. (a) the dependence of four largest clusters $S_{1,2,3,4}$ (solid, dash, dash-dot, short dash lines) on $\sigma_{1}$. (b) the dependence of the relative number of clusters $N_{c} / N$ on $\sigma_{1}$. (c) the dependence of the Shannon information entropy $H$ on $\sigma_{1}$. (d) the relation of $N_{c} / N$ against $S_{1}$. (e) the relation of $N_{c} / N$ against $H$. (f) the relation of $S_{1}$ against $H$. In panels (d-f), the colors of dots for the values of $N$ logarithmically.

strongly connected local structure in SWNs are strongly affected by $N$.

\section{CONCLUSION AND DISCUSSION}

In this work, we studied cluster profiles of a heterogeneous population obeying the modified HegselmannKrause opinion dynamic rule on complex networks. The cluster profile could be described by not only the number of clusters, but also the relative size of the largest cluster, as well as the Shannon information entropy about relative sizes of all clusters. We found the cluster profile was the trade-off of competition between all parameters of this heterogeneous population, including the stubbornness, the bounded confidence, and the subpopulation ratio. Except that even if agents in another subpopulation were open-minded, increasing the bounded confidence could lead to more clusters and a smaller size of the largest cluster on complex networks, which was quite similar with that on complete graphs [14]. Optimal bounded confidence of one subpopulation existed for the fewest clusters, the largest size of largest cluster, and the lowest information gain. While increasing the stubbornness of one subpopulation led to the most clusters and the highest entropy. Increasing the ratio of open-minded agents was not always beneficial to the fewest clusters and the lowest entropy. Moreover, we found that the dependent relations between $H, N_{c}$, and $S_{1}$ were almost independent of network structures when only one of parameters varied, including bounded confidence, stubbornness, and subpopulation ratio.

Furthermore, we also investigated effects of parame- 
ters of network structures on cluster profiles, such as the average degree and the network size. The difference between network structures remained when the average degree was small, while it vanished when the average degree was large enough. The large average degree made networks similar with a complete graph, where cluster profiles on it had a larger $S_{1}$, a smaller $N_{c}$, and a lower $H$. As for the network size, cluster profiles of heterogeneous populations on scale-free networks shared many similar features, while those on small-world networks showed more differences, and those on random networks were in the medium. These different results between different network structures were caused by the strongly connected local structures in small-world networks, hubs-leaf structures in scale-free networks, and random connection structure in random networks. These two findings showed that network structures could affect the evolution of opinion dynamics indeed.

Our work sheds light upon the cluster profile of a heterogeneous population on complex networks. Cluster profiles on different networks have many similar features but also display some differences. However, the conclusion in this work is just from one perspective. It is worthy studying the mechanisms to forge consensus from other perspectives and in detail. For example, it may be interesting to study a heterogeneous population on some special networks, such as networks with symmetry [33].
[1] C. Castellano, S. Fortunato, V. Loreto, "Statistical physics of social dynamics," Review of Modern Physics, vol. 81, no. 2, pp. 591-646, 2009.

[2] Z. Wang, C. T. Bauch, S. Bhattacharyya et al., "Statistical physics of vaccination," Physics Reports, vol. 664, pp. 1-113, 2016.

[3] Serge Galam, "Unavowed abstention can overturn poll predictions," Fronters in Physics, vol. 6, Article ID 24, 2018.

[4] R. Holley, T. Liggett, "Ergodic theorems for weakly interacting infinite systems and the voter model," The Annals of Probability, vol. 3, no. 4 pp. 643-663, 1975.

[5] K. Sznajd-Weron, J. Sznajd, "Opinion evolution in closed community," International Journal of Modern Physics C, vol. 11, no. 6, pp. 1157-1165, 2000.

[6] Serge Galam, "Minority opinion spreading in random geometry," The European Physical Journal B, vol. 25, no. 4, pp. 403-406, 2002.

[7] J. Shao, S. Havlin, H. E. Stanley, "Dynamic opinion model and invasion percolation," Physical Review Letters, vol. 103, no. 1, Article ID 018701, 2009.

[8] H. Yang, L. Huang, "Opinion percolation in structured population," Computer Physics Communications, vol. 192, pp. 129-142, 2015.

[9] G. Deffuant, D. Neau, F. Amblard et al, "Mixing beliefs among interacting agents," Advances in Complex Systems, vol. 03, no. 01n04, pp. 87-98, 2000.

[10] R. Hegselmann, U. Krause, "Opinion dynamics and bounded confidence models, Analysis and Simulation," Journal of Artificial Societies Social Simulation, vol. 5, no. 3, Artical ID 2, 2002.

[11] Jan Lorenz, "Heterogeneous bounds of confidence: Meet, discuss and find consensus!" Complexity, vol. 15, no. 4, pp. 43-52, 2009.

[12] M. Pineda, G. M. Buenda, "Mass media and heterogeneous bounds of confidence in continuous opinion dynamics," Physica A, vol. 420, pp. 73-84, 2015.

[13] G. Fu, W. Zhang, Z. Li, "Opinion dynamics of modified HegselmannCKrause model in a group-based population with heterogeneous bounded confidence," Physica A, vol. 419, pp. 558-565, 2015.

[14] W. Han, C. Huang, J. Yang, "Opinion clusters in a modified Hegselmann-Krause model with heterogeneous bounded confidences and stubbornness," Physica A, vol.
531, Artical ID 121791, 2019.

[15] J. Ghaderi, R. Srikant, "Opinion dynamics in social networks with stubborn agents: Equilibrium and convergence rate," Automatica, vol. 50, no. 12, pp. 3209-3215, 2014.

[16] A. Olshevsky, J. Tsitsiklis, "Convergence speed in distributed consensus and averaging," SIAM Journal on Control and Optimization, vol. 48 no. 1, pp. 33-55, 2009.

[17] B. Chazelle, C. Wang, "Inertial Hegselmann-Krause systems," IEEE Transactions on Automatic Control, vol. 62, no. 8, pp. 3905-3913, 2017.

[18] X. Chen, S. Zhao, W. Li, "Opinion dynamics model based on cognitive styles: Field-dependence and field independence," Complexity, vol. 2019, Artical ID 2864124, 2019.

[19] P. Erdös, A. Rényi, "On the evolution of random graphs," Publ. Math. Inst. Hung. Acad. Sci. vol. 5, pp. 17-60, 1960.

[20] A.-L. Barabási, R. Albert, "Emergence of Scaling in Random Networks," Science, vol. 286, no. 5439, pp. 509-512, 1999.

[21] D. J. Watts, S. H. Strogatz, "Collective dynamics of small-world networks," Nature, vol. 393, no. 6684, pp. 440-442, 1998.

[22] M. A. Porter, J. P. Gleeson, "Dynamical systems on networks: A tutorial," Frontiers in Applied Dynamical Systems: Reviews and Tutorials, Vol. 4, Springer International Publishing Switzerland, 2016.

[23] X. F. Meng, R. A. Van Gorder , M. A. Porter, "Opinion formation and distribution in a bounded-confidence model on various networks," Physical Review E, vol. 97, no. 2, Artical ID 022312, 2018.

[24] O. AskariSichani, M. Jalili, "Influence maximization of informed agents in social networks," Applied Mathematics and Computation, vol. 254, pp. 229-239, 2015.

[25] G. Weisbuch, G. Deffuant, F. Amblard et al., "Meet, discuss, and segregate!" Complexity, vol. 7, no. 3, pp. 55-63, 2002.

[26] Mahdi Jalili, "Social power and opinion formation in complex networks," Physica A, vol. 392, no. 4, pp. 959966, 2013.

[27] Y. Gandica, M. del Castillo-Mussot, G. J. Vzquez et al., "Continuous opinion model in small-world directed networks," Physica A, vol. 389, no. 4, pp. 5864-5870, 2010.

[28] F. Bagnoli, R. Rechtman, "Topological bifurcations in a model society of reasonable contrarians," Physical Re- 
view E, vol. 88, no. 6, Article ID 062914, 2013.

[29] F. Bagnoli, R. Rechtman, "Bifurcations in models of a society of reasonable contrarians and conformists," Physical Review E, vol. 92, no. 4, Article ID 042913, 2015.

[30] S. Wang, L. Rong, J. Wu, "Bistability and multistability in opinion dynamics models," Applied Mathematics and Computation, vol. 289, pp. 388-395, 2016.

[31] C. E. Shannon, "A mathematical theory of communication," The Bell System Technical Journal, vol. 27, no. 3, pp. 379-423, 1948.

[32] Jan Lorenz, "Continuous opinion dynamics under bounded confidence: a survey," International Journal of Modern Physics C, vol. 18, no. 12, pp. 1819-1838, 2007.

[33] I. Klickstein, L. Pecora, F.Sorrentino, "Symmetry induced group consensus," Chaos, vol. 29, no. 7, Artical ID 073101, 2019. 